\title{
Primate mandibular reconstruction with prefabricated, vascularized tissue-engineered bone flaps and recombinant human bone morphogenetic protein-2 implanted in situ
}

\author{
Miao Zhou ${ }^{\text {a,b }}$, Xin Peng ${ }^{\mathrm{a}}$, Chi Mao ${ }^{\mathrm{a}}$, Fang $\mathrm{Xu}^{\mathrm{c}}$, Min Hu ${ }^{\mathrm{d}, * *}$, Guang-yan $\mathrm{Yu}^{\mathrm{a}, *}$ \\ Q1 a Department of Oral and Maxillofacial Surgery, Peking University School and Hospital of Stomatology, Beijing 10081, China \\ ${ }^{\mathrm{b}}$ Department of Oral and Maxillofacial Surgery, The Second Affiliated Hospital of Sun Yat-sen University, Guangzhou, China \\ ${ }^{\mathrm{c}}$ College of Pharmaceutical Science, Zhejiang University of Technology, Hangzhou, China \\ ${ }^{\mathrm{d}}$ Department of Oral and Maxillofacial Surgery, Chinese PLA General Hospital, Beijing, China
}

\section{A R T I C L E I N F O}

\section{Article history:}

Received 16 February 2010

Accepted 28 February 2010

Available online $\mathrm{xxx}$

\section{Keywords:}

Mandibular reconstruction

Prefabricated vascularized bone flaps rhBMP-2

Demineralized freeze-dried bone allograft Coralline hydroxyapatite

\section{Introduction}

The restoration of mandibular defects caused by ablative surgery for oral and maxillofacial tumor, trauma, infection and congenital deformity remains a challenge for surgeons [1]. Autol ogous grafting and distraction osteogenesis are the most common techniques used to restore mandibular defects [2]. Autologous grafts have been considered the "gold standard" because of the advantages of osteogenesis, osteoinduction and osteoconduction.

\footnotetext{
* Corresponding author. Tel.: +86 10 62191099; fax: +86 1062173402 .

** Corresponding author.

E-mail address: gyyu@263.net (G.-y. Yu).
}

\begin{abstract}
A B S T R A C T
Several studies have validated successful mandibular reconstruction with prefabricated tissue engi neered bone flaps and recombinant human bone morphogenetic protein 2 (rhBMP 2) implanted in situ. Whether rhBMP 2 applied with the prefabrication technique enables faster ossification of mandibular defects than rhBMP 2 applied in situ is unknown. We aimed to compare mandibular reconstruction with prefabricated, vascularized tissue engineered bone flaps with rhBMP 2 and rhBMP 2 applied in situ in primates (Rhesus monkey). We also compared the use of the carriers demineralized freeze dried bone allograft (DFDBA) and coralline hydroxyapatite (CHA) for applying rhBMP 2. After computed tomography of the monkey head, custom meshes were made, loaded with rhBMP 2 incorporated DFDBA or CHA, and implanted in the latissimus dorsi muscle. Meanwhile, contralateral segmental mandibular defects were created, and custom meshes loaded with DFDBA, CHA, or rhBMP 2 incooperated DFDBA and CHA were implanted in situ. Thirteen weeks later, the bone flaps with rhBMP 2 incorporated DFDBA or CHA were transferred to repair segmental mandibular defects. The meshes loaded with DFDBA or CHA alone showed no bone regeneration 13 weeks after implantation in latissimus dorsi muscle. Radiography, angiography and histological analysis were used to evaluate the repair and vascularization of the implant. Segmental mandibular defects were successfully restored with prefabricated bone flaps and rhBMP 2 incorporated CHA in situ, but other segmental mandibular defects remained with rhBMP 2 incorporated DFDBA, DFDBA and CHA in situ. Moreover, mandibles reconstructed with rhBMP 2 incorporated CHA bone flaps revealed more regenerated and homogeneous bone formation than did other reconstructions. The study suggested that the prefabrication technique induced better mandibular reconstruction and bone regeneration in quantity and quality.
\end{abstract}

(c) 2010 Elsevier Ltd. All rights reserved.

0142-9612/\$ see front matter (c) 2010 Elsevier Ltd. All rights reserved. doi:10.1016/j.biomaterials.2010.02.072 
tissues for restoring large compound mandibular defects with preceding radiotherapy and scars in situ. Animal studies also vali dated the effectiveness of the 2 methods in mandibular recon struction [11-13]. Whether the prefabrication technique enables faster ossification than with recombinant human bone morpho genetic proteins (rhBMPs) implanted in situ is still unknown, especially for large compound mandibular defects.

Carriers are used to increase the retention of rhBMPs at ortho paedic treatment sites for a sufficient period of time to allow regenerative tissue to form cells that migrate to the area of injury and to proliferate and differentiate [14]. The four major categories of carrier materials for rhBMPs include natural polymers, inorganic materials, synthetic polymers and composites of these materials [15]. Demineralized freeze dried bone allograft (DFDBA) and coralline hydroxyapatite (CHA) have been studied as carriers for rhBMP 2. Clokie et al. reconstructed 10 mandibular defects with a construct containing osteogenetic protein 1 (OP 1 ) in a deminer alized bone matrix suspended in a reverse phase medium to effect sustained rhBMPs delivery [9]. Heliotis et al. reconstructed a human mandibular defect with a prefabricated tissue engineered bone flap by implanting OP 1 coated CHA in the pectoralis major muscle [10]. However, none of the research compared the ossification with ectopic or orthotopic rhBMP 2 incorporated DFDBA or CHA carriers.

We aimed to compare the effectiveness of rhBMP 2 applied in situ and applied in muscle of a prefabricated, vascularized tissue engineered bone flap for mandibular reconstruction. DFDBA and CHA were also evaluated as constructs in mandibular reconstruction.

\section{Materials and methods}

\subsection{Manufacture and specification of DFDBA and CHA}

DFDBA was made from the control group of a pharmacokinetics study of recombinant human stem cell growth factors in Rhesus monkeys (Beijing Joinn Laboratories, China). The donor Rhesus monkeys were healthy and 68 years old. Briefly, the spongy bone was obtained from the extremities and reserved at $-70{ }^{\circ} \mathrm{C}$ for 2 months to extinguish immunogenicity. The spongy bone was separated from the head of extremities and cut into 2 sizes: scaffold " $A$ " with upper plane $10 \mathrm{~mm} \times$ $3 \mathrm{~mm}$, lower plane $10 \mathrm{~mm} \times 6 \mathrm{~mm}$ and height $5 \mathrm{~mm}$; and scaffold "B" with size 10 $\mathrm{mm} \times 6 \mathrm{~mm} \times 5 \mathrm{~mm}$. The bone blocks were then soaked in acetone for $48 \mathrm{hr}$ to remove the fatty composition. Distilled water was used to wash out bone marrow and rudimentary blood, then the bone block sample was demineralized with $0.6 \mathrm{~m}$ HCL and washed with distilled water, then freeze-dried. The CHA scaffold was provided by Yihuajian Commercial Co. Ltd. (China). It was manufactured as for the DFDBA scaffold. Then, the samples were prepared by hydrothermal chemical exchange with phosphate as per the manufacturer's protocol (Yihuajian Commercial Co. Ltd.). The porous morphology of the carriers was analyzed by scanning electron microscopy (SEM, JOEL, Model JSM-5600LV). The sample was freeze-dried and prepared for rhBMP-2 incorporation.

\section{2. rhBMP-2 delivery}

rhBMP-2 was produced by recombinant expression in Escherichia coli at the Genetic Institute of Huadong Medicine (China) and purified to more than $98 \%$ Hangzhou Future Biotech Co. Ltd. (China) supplied the rhBMP-2 used in the experiment. rhBMP-2 $(72 \mathrm{mg}$ ) was dissolved in gelatin solution in acetic acid ( $30 \mathrm{mg} / \mathrm{ml})$. One block of each carrier was soaked in $1 \mathrm{ml}$ gelatin solution with rhBMP-2 (1.5 $\mathrm{mg} / \mathrm{ml}$ ) (DFDBA-BMP and CHA-BMP, respectively). rhBMP-2-incorporated scaffolds were freeze-dried in frozen tubes and kept at $27{ }^{\circ} \mathrm{C}$ until use.

DFDBA and CHA scaffolds coated with gelatin but without rhBMP-2 were also prepared. Particles of CHA and DFDBA (size: $0.41 .0 \mathrm{~mm}$ ) were prepared.

\subsection{Experimental design}

Nine adult male healthy Rhesus monkeys ( 69 years old, $612 \mathrm{~kg}$ ) were provided by the Laboratory Animal Center of the Chinese PLA General Hospital (China). Surgical techniques and animal care conformed to the principles of the Laboratory Animal Center (NIH publication No. 85-23, revised 1985). For prefabricating tissueengineered bone flaps, custom meshes loaded with DFDBA-BMP ( $n$ 3), CHA-BMP ( $n$ $3)$, DFDBA ( $n$ 3) and CHA ( $n$ 3) were implanted in animals' ambilateral latissimus dorsi muscle. Thirteen weeks later, ossification was validated in the groups of DFDBA-BMP and CHA-BMP, while no ossification was found in the other groups. Then, the prefabricated tissue-engineered bone flaps with ossification were transferred to repair homolateral segmental mandibular defects. At the time of surgical implantation in the latissimus dorsi muscle, contralateral segmental mandibular defects described below were implanted with DFDBA-BMP, CHA-BMP, DFDBA and CHA respectively.

The bilateral mandibular defects in 9 monkeys were randomized into 6 groups for treatment: P-DFDBA-BMP and P-CHA-BMP: mandibular defect repaired by prefabricated tissue-engineered bone flap with DFDBA-BMP and CHA-BMP, respectively (n 3 each); S-DFDBA-BMP and S-CHA-BMP: mandibular defect repaired by in situ DFDBA-BMP and CHA-BMP, respectively ( $n 3$ each); and S-DFDBA and S-CHA: mandibular defect repaired by in situ DFDBA and CHA, respectively ( $n \quad 3$ each). The study was approved by the Animal Care and Experiment Committee of Peking University Health Science Center.

\subsection{Manufacture of custom titanium mesh}

3-D computed tomography (CT) images were taken of the heads of monkeys The scanning data were uploaded to a computer-aided design program, 3-D medicine surface rendering (3DMSR, JIMAFEI Science and Technology Development Co. Ltd., China) software system. A 20-mm-long mandibulectomy was virtually created in the mandibular body (Fig. 1a and b). Then, the data were transferred to a milling machine (GSVM6540, Gold Sun Mould \& CNC machinery Co. Ltd., China). The custom titanium meshes for internal fixation of the stumps of the mandible were made by the machine. The meshes were used to shape the tissue-engineered bone in the latissimus dorsi muscle and internal fixation of mandibular stumps. The buccal and lingual height of the mesh was 12 and $10 \mathrm{~mm}$, respectively (Fig. 1c and d).

\subsection{Surgical procedures}

General anesthesia was induced with ketamine hydrochloride $(20 \mathrm{mg} / \mathrm{kg}$ intramuscularly or subcutaneously) and maintained with pentobarbital sodium (1 $2 \%$, intravenously). The monkeys were prepared for extraction of mandibular teeth from the bicuspid tooth to the back molar. Three months after extraction, the wounds of the socket healed for the next segmental mandibulectomy.

With monkeys under general anesthesia, the latissimus dorsi muscle was visualized through a lateral approach, and a pouch was made inside the muscle close to the thoracodorsal artery. The custom mesh was loaded with 4 blocks of scaffold (containing $2 \mathrm{~A}$ and B, respectively). The particles of DFDBA or CHA scaffolds were mixed with the blood from the surgery. Then the mixture was used to fill the gap between the block and the custom mesh. The pre-loaded titanium mesh was implanted into the pouch of the latissimus dorsi muscle of anesthetized monkeys (Fig. 2a c). Thirteen weeks after implantation, the flap was harvested along with the adjoining part of the latissimus dorsi muscle containing the thoracodorsal artery and vein, which supplied blood to the bone flap. The bone flap was biopsied (Fig. 2d). The segmental mandibular defect was carried out as follows. After a sub-mandibular incision, the mandible was exposed extra-orally through a full-thickness flap that included the periosteum. A 20-mm-wide section of the mandible from the bicuspid tooth to the back molar teeth was resected with a fissure bur (Fig. 2e). The volume of the defect was approximately $3 \mathrm{~cm}^{3}(20 \mathrm{~mm} \times 10 \mathrm{~mm} \times 15 \mathrm{~mm})$. The wound of the defect was irrigated with normal saline. The prefabricated tissue-engineered bone flap was then transferred to the mandibular defect via a tunnel created beneath the major pectoral muscle (Fig. $2 \mathrm{f}$ ). For study the bone regeneration with rhBMPs or scaffold alone implanted in situ, the titanium mesh loaded with DFDBA-BMP, CHABMP, DFDBA or CHA was fixed onto the stumps of the contralateral mandibular defects with titanium micro-osteosynthesis screws.

\subsection{Clinical view, angiography and radiography examinations}

Postoperative activities, emotional response, food intake and would healing of surgical sites were observed in all animals. The status of bone regeneration of the custom meshes loaded with DFDBA-BMP and CHA-BMP were examined during transplantation. At 26 weeks after implantation, animals were sacrificed by intravenous overdose of sodium pentobarbitone, and mandibles and loaded custom meshes were excised. In 2 Rhesus monkeys, the lingual side of the mesh was integrated with the regenerated bone and was saved.

Mandibular radiogram was obtained (X-mind AC, ACTEON, France). The area of the bone regenerated in the mandibular defect was analyzed by the Leica image analysis system.

Angiography was performed before sacrifice in 4 animals to validate the blood supply of the prefabricated tissue-engineered bone flap. The latissimus dorsi muscles containing the titanium meshes were dissected. Two veins and 1 artery were in the thoracodorsal vascular bundle. One thoracodorsal artery was cannulated. Subsequently, the graft was perfused at $37{ }^{\circ} \mathrm{C}$ with $130 \mathrm{mmHg}$ pressure and $100 \mathrm{ml}$ of $49 \mathrm{ml}$ normal saline, $50 \mathrm{ml}$ Ultra-vist370 (Bayer Schering Pharma. Germany) and 10000 U heparin. In 2 Rhesus monkeys, angiography of the distal end of the thoracodorsal artery was performed to validate the relation of the thoracodorsal artery to the transferred tissue-engineered bone flap. 

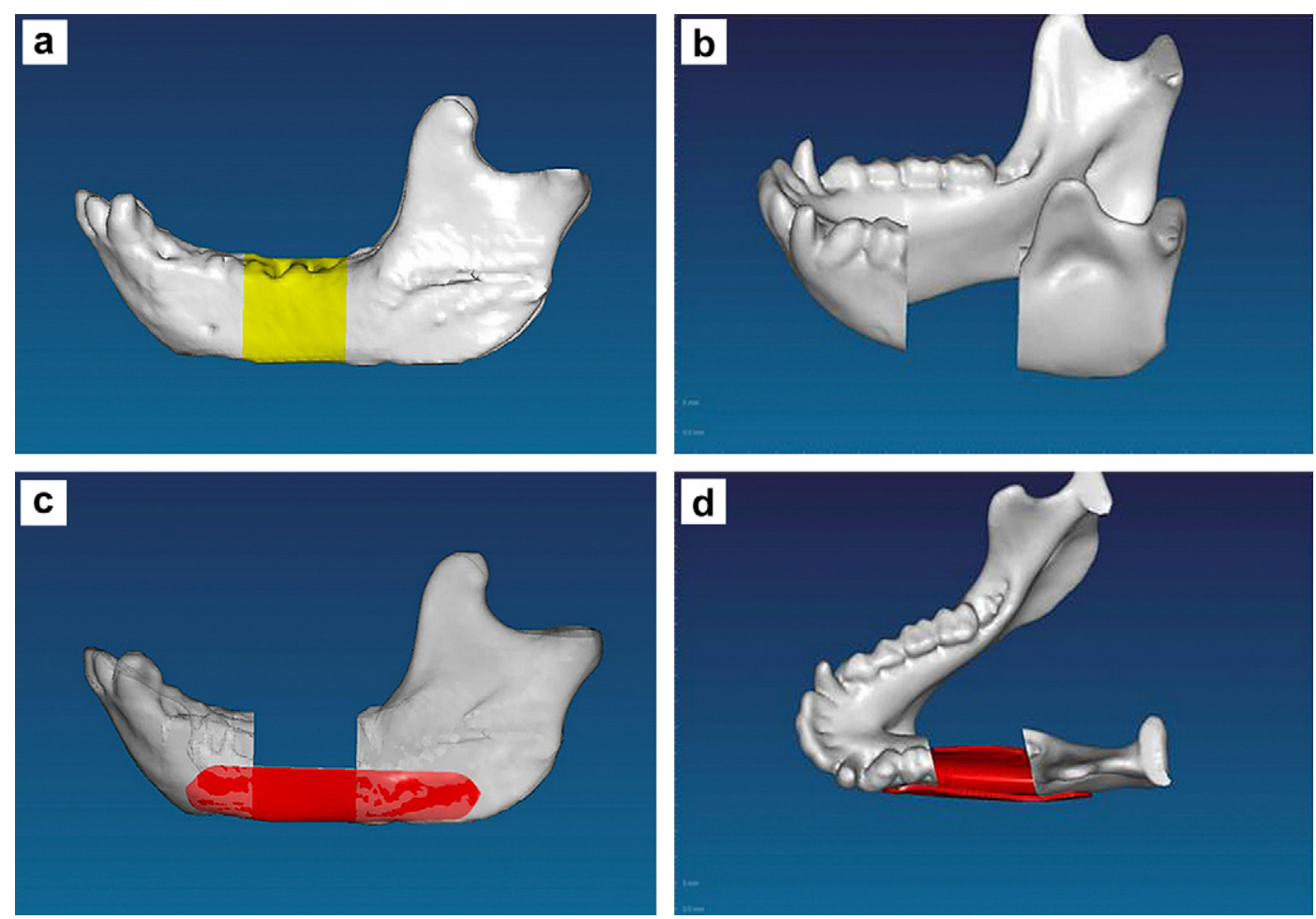

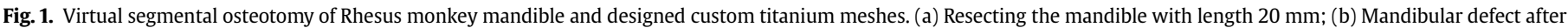
virtual osteotomy; (c) Lateral view of the mesh on the outer surface of the mandible; (d) Occlusional view of the mesh.

\subsection{Histological analysis}

For simultaneous sequential intravital staining of the regenerated bone, intraperitoneal injection of fluorochromes began at 2 weeks after implantation with the custom meshes. Alizarin-complexion ( $3 \%$ in $2 \% \mathrm{NaHCO}_{3}$ solution, $0.8 \mathrm{ml} / \mathrm{kg}$ body weight) was injected 2 and 4 weeks after implantation. Tetracycline (1\% in normal saline, 1 $\mathrm{ml} / \mathrm{kg}$ body weight) was injected 8 and 10 weeks after implantation. Xylenol orange (6\% in $2 \% \mathrm{NaHCO}_{3}$ solution, $1.5 \mathrm{ml} / \mathrm{kg}$ body weight) was injected 14 and 18 weeks after implantation. Calcein ( $1 \%$ in $2 \% \mathrm{NaHCO}_{3}$ solution, $5 \mathrm{ml} / \mathrm{kg}$ body weight) was injected 22 and 24 weeks after implantation (all fluorochromes from Sigma).

Mandibular biopsy specimens taken during surgery were fixed in $10 \%$ neutral buffered formalin. After dehydration, specimens were immersed in Technovit 7200VLC (Heraeus-Kulzer, Germany). After solidification for $24 \mathrm{hr}$, specimens were cut from the longitude of mandibles. Tissues with P-CHA and P-DFDBA inside the titanium meshes were separated for histological examination. Histological preparation was previously described [16]. The embedded samples were cut into thin sections approximately $4080 \mu \mathrm{m}$, and mounted on glass slides. Three ground sections from each specimen block, $300 \mu \mathrm{m}$ apart, were stained with haematoxylin and eosin (H\&E). Histomorphometry involved the Leica image analysis system.

\subsection{Statistical analysis}

Data from radiography and histology are expressed as mean \pm s.e.m and were analyzed by one-way ANOVA. The differences between 2 groups among the 6 groups were assessed with the Student Newman Kewls test. $P<0.05$ was considered statistically significant.

\section{Results}

\subsection{Properties of DFDBA and CHA}

To study the retention of ectopic and orthotopically adminis tered rhBMP 2, we chosed the two classical carriers for rhBMPs, DFDBA and CHA. The DFDBA prepared from the extremities of rhesus monkeys was considered an allograft. The porous structure of DFDBA enabled rhBMP 2 to infiltrate deeply into the carrier and release slowly after implantation. SEM of DFDBA revealed no osteoblast or crystal structure on the scaffold surface. The mean size of the pores in the trabecular zone was larger than that in cortical bone $(400-600 \mu \mathrm{m}$ [Fig. 3a] and $100-200 \mu \mathrm{m}$, respec tively). SEM of CHA revealed a porous structure. The pore size of CHA was $270-550 \mu \mathrm{m}$ (Fig. 3b), which was similar to the structure of human trabecular bone. Many hydroxyapatite crystals adhered to the surface. The porous structure enables blood capillary sprout, nutrient diffusion and bone ingrowth to the scaffold.

\subsection{Gross view, angiography and radiography examinations}

All wounds healed. No sign of flap necrosis was visible during the postoperative period. Postoperative swelling was noted in all animals. The swelling of the latissimus dorsi muscle with DFDBA BMP and CHA BMP implants lasted longer than that with DFDBA or CHA implants alone. The titanium meshes loaded with DFDBA BMP and CHA BMP showed ossification inside the mesh. No overgrowth of the regenerated bone was found outside the mesh, and no osteointegration with the titanium meshes occurred. Meshes loaded with DFDBA BMP or CHA BMP were encapsulated by the latissimus dorsi muscle. The volume of the bone regenerated was larger with CHA BMP than DFDBA BMP loading. No bone induction was found in meshes loaded with DFDBA, and the inner space of the meshes was occupied by the latissimus dorsi muscle. With the CHA loading, nearly $50 \%$ of the implant had disappeared, and the rest of CHA was the block encapsulated by the latissimus dorsi muscle (data not shown).

Angiography was used to study the blood supply with trans ferred tissue engineered bone flaps and validate the relation of the thoracodorsal bundle to the implant. The transferred thoracodorsal bundle was located from the axillary fossa to the repaired mandibular defect, which validated that the transferred tissue engineered bone flap was supplied by the thoracodorsal artery and vein (Fig. 4a). Thoracodorsal arteries had one main trunk and 2-4 branches. The thoracodorsal vein had 2 trunks. Angiography also 

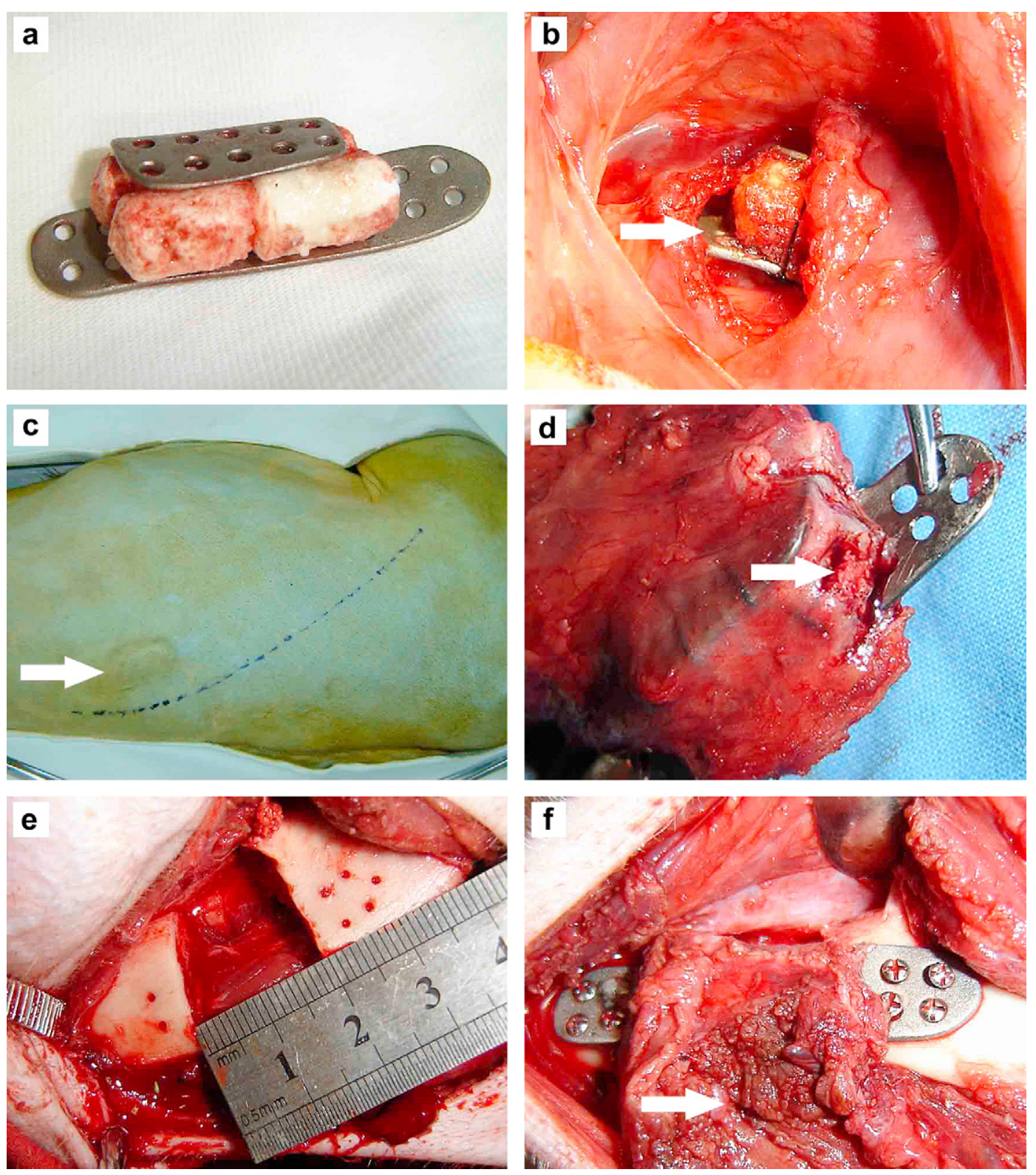

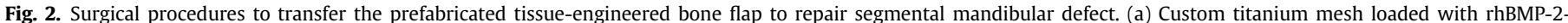

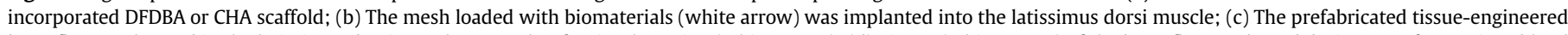

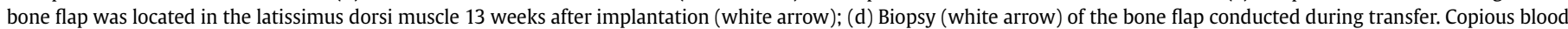

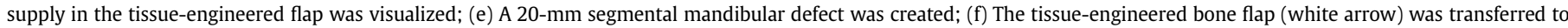
reconstruct the segmental mandibular defect.

validated the existence of a copious vascular net in the latissimus dorsi muscle, which enabled faster vascularization of the tissue engineered bone flaps (Fig. 4b).

Lateral and vertical radiography was used to evaluate bone regeneration and healing in the mandibular defect. With P DFDBA BMP implants, the height of the regenerated bone was comparable to that of the adjacent mandible. The width of the regenerated bone was less than that of normal mandible. Mandibular defects were reconstructed successfully. The block of DFDBA was invisible. The boundary between the regenerated bone and the adjacent mandible had disappeared. With P CHA BMP implants, the height and shape of the bone regenerated was similar to that of the adjacent mandible. The block of CHA was partly absorbed. The gap between the CHA blocks was filled by newly formed bone. The mandibular defect was fully repaired. With S DFDBA BMP implants, the blocks of DFDBA had disappeared. Half of the mandibular defects were occupied by newly formed bone. The discontinuity of the mandible remained. With S CHA BMP implants, the CHA blocks were partly absorbed. Bone defects were apparent in the buccal side of regenerated bone. The mandibular continuity was restored. With S DFDBA and S CHA implants, the mandibular discontinuity was remained. With S DFDBA implants, the DFDBA blocks were invisible in the defect. The CHA blocks showed a uniformly higher radiopacity in the mandibular defect with P CHA BMP and S CHA BMP than S CHA implants, which validated the bone ingrowth to the carrier. The lingual side of the mandibular defect showed homogeneous bone regeneration induced by the lingual periosteum (Fig. 5).

In agreement with the radiography examination, the gross view of mandibular reconstruction showed perfect bone union with $\mathrm{P}$ 

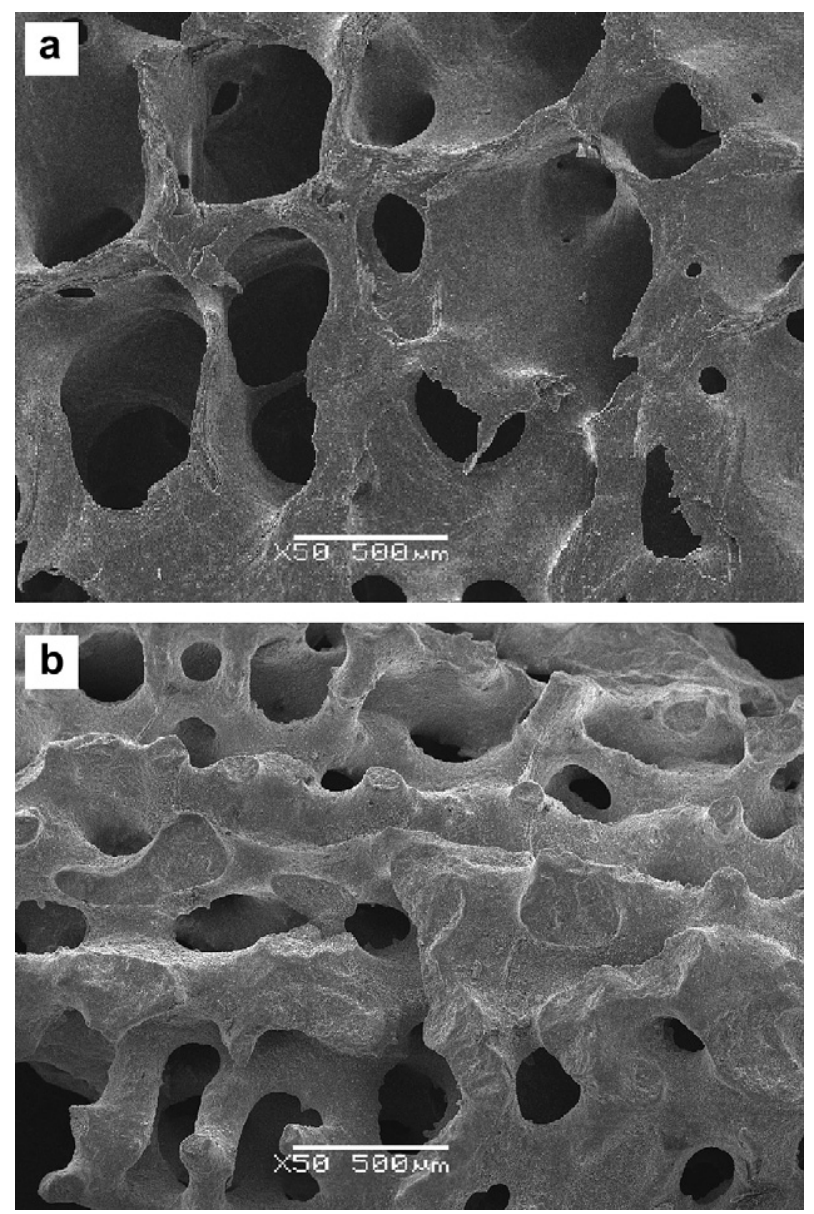

Fig. 3. Scanning electronic microscopy examination of DFDBA and CHA scaffolds. 3-D porous structure of (a) DFDBA and (b) CHA.

CHA BMP implants at 26 weeks after implantation. The lingual side of the reconstructed bone showed bone overgrowth around the titanium mesh. With P DFDBA BMP, P CHA BMP and S CHA BMP implants, bone defects were revealed in buccal side of the custom mesh. Soft tissue appeared in the gap between the regenerated bone and the buccal side of the mesh. Mandibular defects were well reconstructed with $\mathrm{P}$ DFDBA BMP, P CHA BMP and S CHA BMP implants but were poorly reconstructed with S DFDBA BMP, $S$ CHA, and S DFDBA implants. With S DFDBA BMP implants, half of the area of the defect was occupied by bone regenerated from the 2 ends to the center. The center of the defect was occupied by soft tissues (data not shown).

\subsection{Histology}

Fluorescent labeling was detected in the regenerated bone in all specimens except for those with S DFDBA and S CHA implants. Alizarin complexion was absent from all groups. Labeling with tetracycline, xylenol orange and calcein revealed continual bone deposition. In the pores of the scaffold, bone label sequences revealed the direction of bone deposition. Bone remodeling was validated by interrupted bone labeling and new bone deposition (Fig. 6).

Histological examination of the prefabricated tissue engineered bone flap in the latissimus dorsi muscle showed rigorous bone regeneration around the carrier. P DFDBA BMP implants showed the formation of mature bone, large bone trabecula, bone marrow and a small area of unabsorbed DFDBA. The osteoblasts lined the regenerated trabecula. P CHA BMP implants showed bone marrow predominantly present in the interconnecting pores of CHA. New bone formed on the surface of the CHA. The morphology of CHA did not change greatly. With P DFDBA implants, the inner part of the mesh was occupied by soft tissue, and no bone formed. Most of the DFDBA implanted in the mesh was resorbed. With P CHA implants, no bone was induced. The meshes were occupied by muscles encapsulating the CHA block (data not shown).

Photomicrographs of histological slices of the mandible are shown in Fig. 7. With P DFDBA BMP, P CHA BMP and S CHA BMP implants, the mandibular defects were reconstructed successfully. The area of calcification with regenerated bone showed interposition of islands of the latissimus dorsi muscle (data not shown). The outside of the meshes was partly osteointegrated with the regenerated bone. The inside of the meshes did not osteointegrate with the transferred tissue engineered bone. The height and volume of the regenerated mandible was comparable in P CHA BMP and S CHA BMP implants. The area of bone regenerated in the mandibular defect was greater with P CHA BMP than P DFDBA BMP implants. The bone regen erated was greater with $P$ CHA BMP than $S$ CHA BMP implants. The bone regenerated with S DFDBA BMP, S DFDBA and S CHA implants was too limited to reconstruct the continuity.
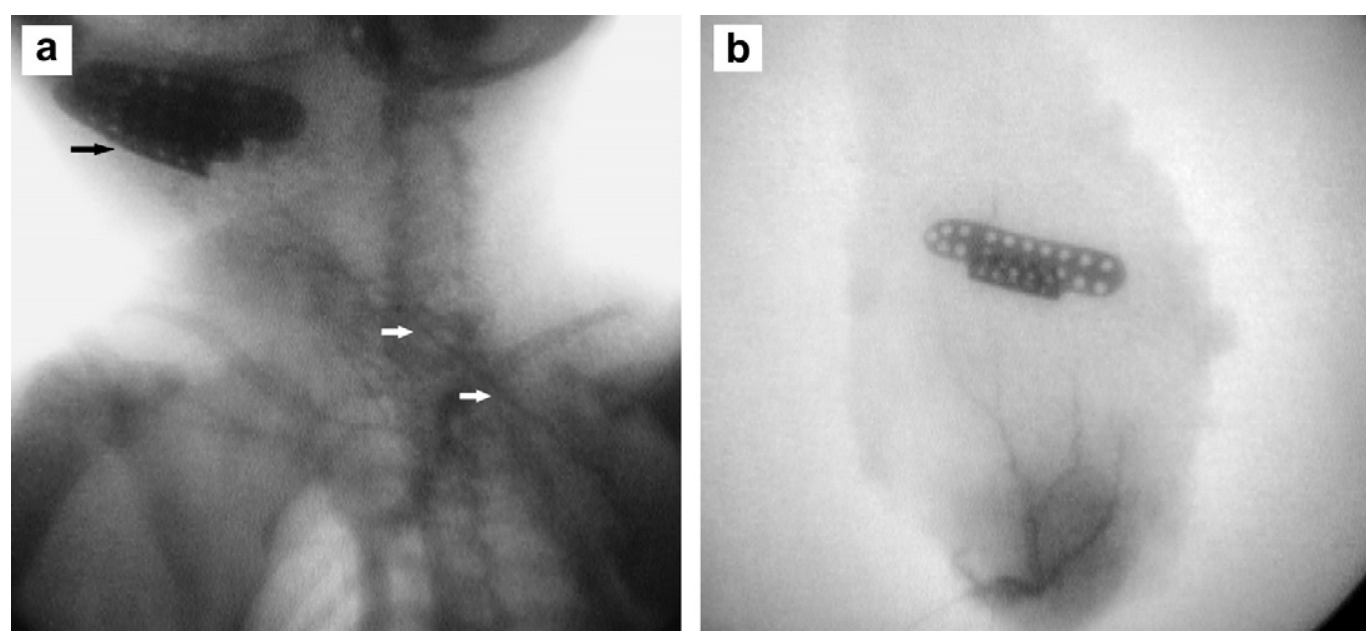

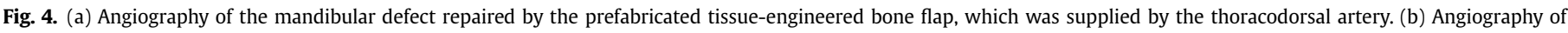
copious blood supply of the bone flap located in the latissimus dorsi muscle. 

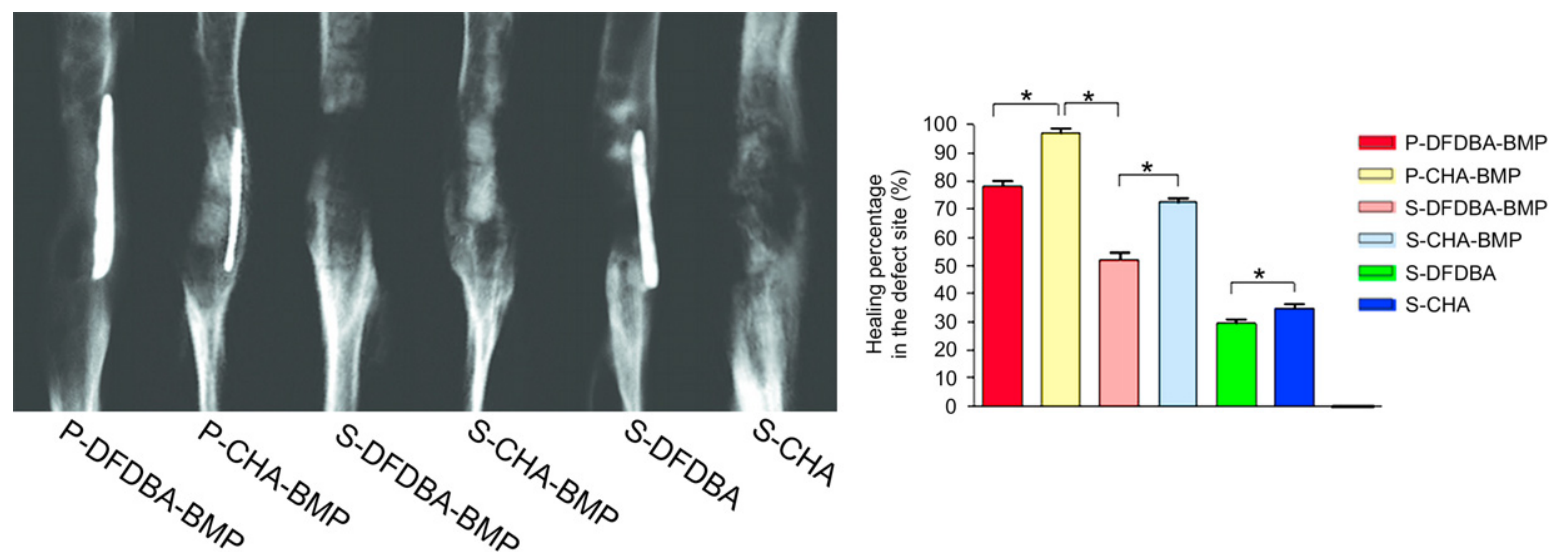

Fig. 5. Radiography of the mandibles reconstructed by the prefabricated tissue-engineered bone flaps or carriers with or without rhBMP- 2 incorporated $\left({ }^{*} P<0.05\right)$.

\section{Discussion}

This study demonstrated successful mandibular reconstruction with prefabricated, vascularized tissue engineered bone flaps and rhBMP 2 implanted in situ. We have compared the techniques of prefabrication vascularized tissue engineered bone flaps in vivo and applying rhBMP 2 in situ for mandibular reconstruction. DFDBA and CHA are known as bone conductive biomaterials for repairing bone defects. In our study, the segmental mandibular defects were similar to those commonly occurring in ablative surgery for malignant tumors in oral and maxillofacial region. These segmental mandibular defects could be restored with rhBMP 2 incorporated CHA scaffolds implanted in situ and not with DFDBA and CHA implanted in situ alone. Therefore, use of rhBMP 2 incorporation is effective in improving the osteoinduc tivity of DFDBA and CHA scaffolds.

At present, tissue engineering is compromised by the inability to adequately vascularize tissues in vivo or in vitro. Vascularization is one of the hurdles in constructing large bone for clinical application, especially for delivering nutrients and removing the degradation product from biodegradable products [17]. Insufficient vascularization can lead to slow bone growth and cell death. Several approaches have achieved initial success in accelerating the vascularization of the construct in vivo or in vitro; examples include scaffold design [18], coculture with endothelial cells (ECs) [19], angiogenic factor delivery [20] and prefabrication in vivo [4]. The method of prevascularization in vitro takes several days to weeks for the center of the implant to be perfused. In addition, in vitro prevascularized constructs have to undergo a process of vessels from the host anastomosing to the existing vasculature [17]. In our study, prevascularization in vivo could induce bone regeneration adjoining the major vessel. As a result, the construct is naturally connected to the host vasculature. Fast, mature and stable vascu larization and bone regeneration can be achieved with this method. As well, the quantity and quality of bone regeneration with prefabrication exceeded that with rhBMP 2 implanted in situ alone. The in vivo prefabrication technique can greatly decrease the time of vascularization in vitro. The drawbacks of this in vivo
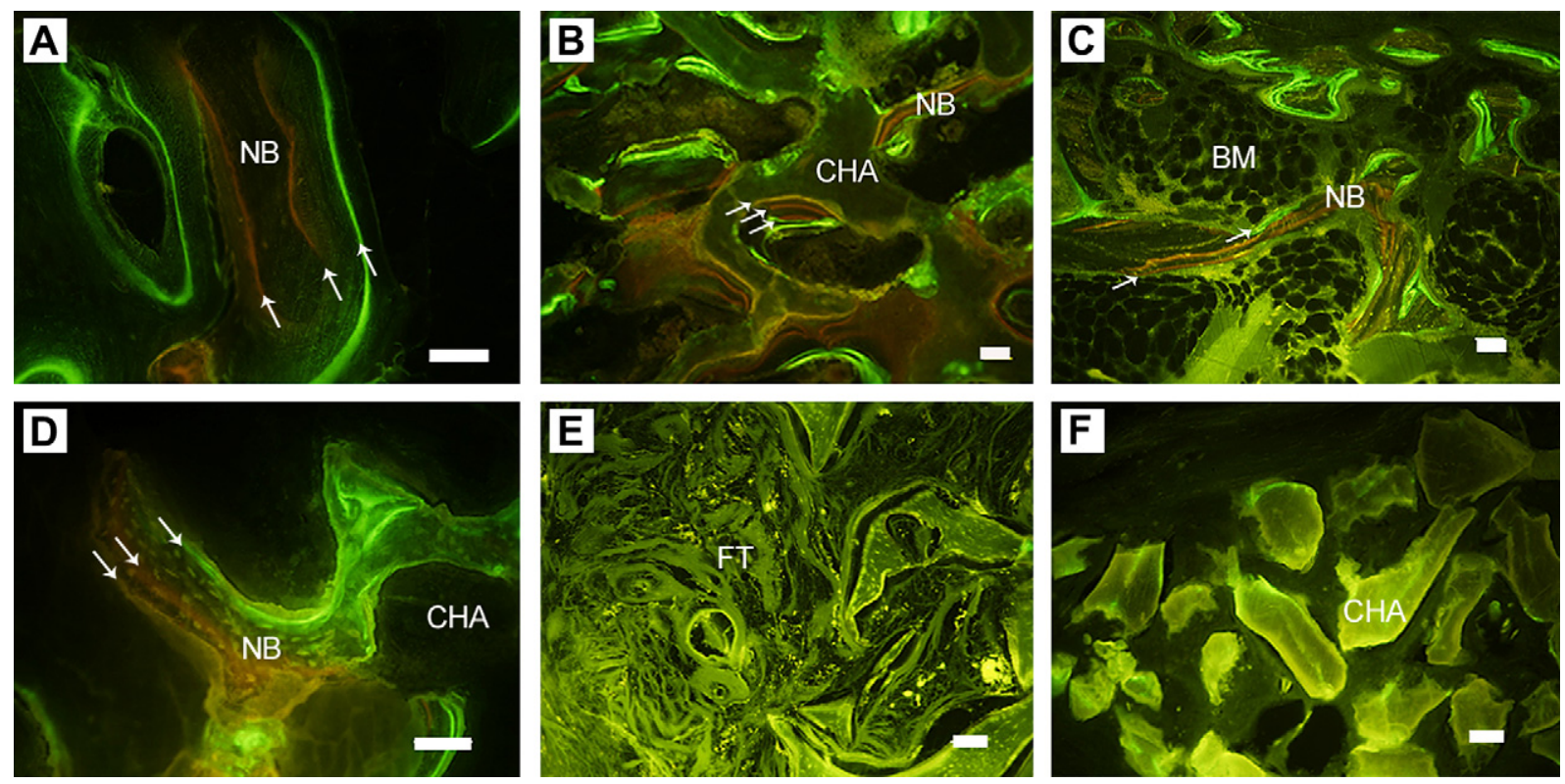

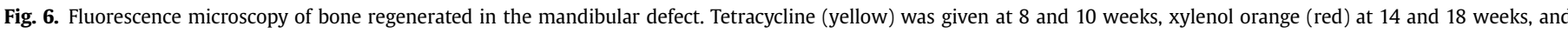

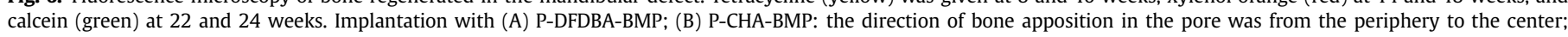

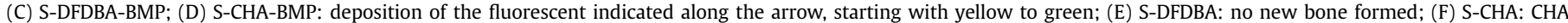

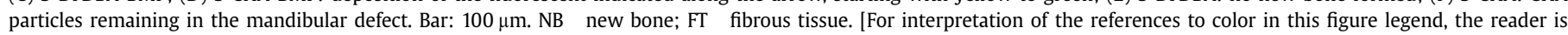
referred to the web version of this article.] 

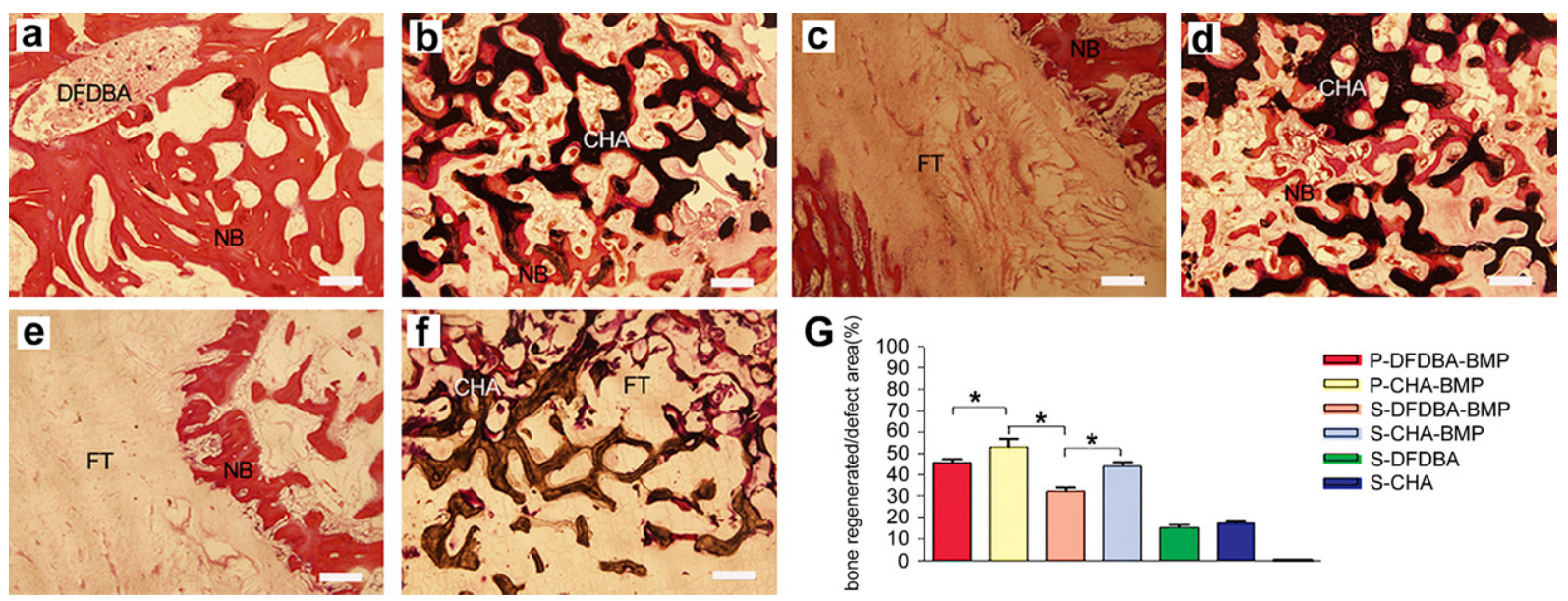

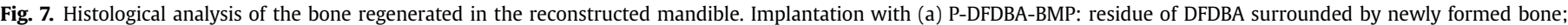

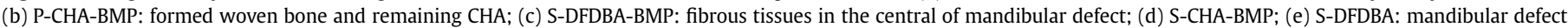

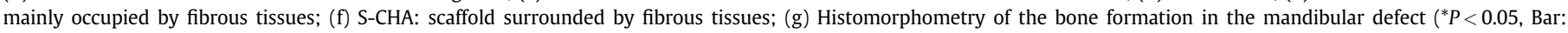
$500 \mu \mathrm{m})$.

prefabrication technique were the inconvenience of two surgical interventions, a rather longer period in ectopic place and donor site morbidity in sacrificing the attached muscle [21].

rhBMPs belong to the superfamily of transforming growth factor $\beta$ and play a important role in embryonic development, including brain and bone formation [22,23]. rhBMP 2 induces bone formation by stimulating mesenchymal stem cells (MSCs) differentiation to osteoblasts. Several studies also revealed successful reconstruction of mandibular defects by rhBMP 2 implanted in situ [8,24]. Martine et al. showed that BMPs stimulate angiogenesis through the production of vascular endothelial growth factor A by osteoblasts [25]. In our study, we combined the strategy of vascularization with rhBMP 2 delivery and implantation with prefabricated bone flaps in vivo to accelerate the vascularization and bone regeneration of the tissue engineered bone. rhBMP 2 accelerated the process of capil lary ingrowth from the muscle to the scaffold. The pedicle of the prevascularized tissue engineered bone flap enabled a more invasive surgical procedure and stable blood supply than with common surgical anastomosis in free transplantation $[4,26]$.

rhBMPs do not remain at therapeutic sites because of rapid diffusion. Different slow releasing systems and carriers were developed to control the dispersion of BMPs from the implant site [14]. An ideal carrier for rhBMPs should be able to maintain a space or volume for bone regeneration and should be biocompatible, absorbable, cost effective, and easily manufactured. However, no favorable carrier has been developed. DFDBA has been widely studied as a carrier for BMPs [27]. CHA is a porous calcium carbonate scaffold coated by hydroxyapatite. The main component of coral is calcium carbonate. The hydroxyapatite coating of CHA can reduce the resorption of the biomaterials. Therefore, CHA is a good osteoconductive scaffold for bone growth. The porous structure of DFDBA and CHA provides an ideal site for MSCs to adhere, proliferate and differentiate. We used gelatin to maintain the slow release of the rhBMP 2 in vivo. CHA showed a better slow release property than did DFDBA. The possible immunologic rejection and quick resorption of DFDBA may impede the slow release of rhBMP 2. Therefore, as a carrier for rhBMP 2, CHA was more stable and effective than DFDBA.

The main component of DFDBA is insoluble, highly cross linked type I collagen. By defatting and demineralization, natural BMP adhering to the surface of collagen is exposed, which increases the osteoinduction of DFDBA. Some studies showed that the osteoinduction of BMP incorporated DFDBA was more stable, effec tive, and longer than that of DFDBA alone [28]. Aspenberg et al. reported on osteoinduction of DFDBA in rat, mouse and rabbit but not in monkey, other primates and even humans [28-30]. Paul et al. found no osteoinduction of DFDBA in human non orthotopic places [31]. DFDBA from young and adult monkeys showed no osteoin ductive property in adult monkey muscle, but, rather, induced bone formation in athymic rat [30]. In our studies, most DFDBA implanted in latissimus dorsi muscle and mandibular defects was resorbed, and no bone regeneration was observed in the latissimus dorsi muscle. In contrast to the results observed in small experimental animals [30], the osteoinductivity of DFDBA is decreased in higher species.

We used custom titanium meshes to shape the regenerated bone. The custom tissue engineered bone flap could be shaped to the mandibular defects for better $3 \mathrm{D}$ outcome. The flanges of the custom mesh were used to fix the regenerated bone to the stump of the mandible. The hole in the mesh engaged the growth of blood vessel from the latissimus dorsi muscle to the regenerated bone. To avoid exposure of the titanium mesh to the oral cavity, the height of the titanium mesh was less than that of the resected mandible. The lingual side of the custom titanium mesh was integrated with the regenerated bone but not in the buccal side. Injury of the buccal periosteum in the mandibular defect may impede the bone regeneration.

Our angiography results showed the blood of the transferred tissue engineered bone flap was supplied by thoracodorsal artery. Bone fluorescence labeling of the transferred bone flap validated the viability and remodeling of the regenerated bone. The amount of bone regenerated in the reconstructed mandible was larger with implantation of prefabricated bone flaps than with that of rhBMP 2 or scaffolds in situ. Terheyden et al. compared direct application of OP 1 and prefabricated vascularized bone grafts in the latissimus dorsi muscle in miniature pigs with mandibular defects [12]. The authors directly implanted OP 1 incorporated BioOss ${ }^{\circledR}$ fixed by absorbable suture, and the critical size defect was in the mandib ular angle, and the internal fixation of the implant differed from our study. The authors found the similar, good results we did with the prefabrication technique. The prefabrication technique may be preferable because the blood supply of muscles is richer than where vascular bed is impaired and muscle provides a stable source of MSCs for cell aggregation and differentiation.

Mandibular defects after ablative surgery of malignant tumor in oral maxillofacial region, especially in the cases with preoperative 
radiotherapy or advanced recurrent carcinoma, usually show compromised blood supply, short of soft tissues and tissue scarring. A successful mandibular reconstruction restores both the shape and function of the mandible, including the contour of the jaw and its surrounding soft tissues, which minimizes the aesthetic defor mity and restores mastication. A free composite bone flap is usually applied in these cases. However, application of this flap is compromised in some patients because of poor anatomic condition in the donor site and radiotherapy, for example. The prefabricated tissue engineered bone flap may be a choice for these composite mandibular defects. In our study, the prefabricated tissue engi neered bone flap showed more stable bone regeneration than with scaffold and rhBMP 2 application alone. When necessary, a composite musculocutaneous flap with tissue engineered bone may be designed to reconstruct a large, mandibular defect with soft tissue loss. This technique can be used as an alternative to vascu larized autologous bone flap.

We used a primate model to apply the 2 techniques of pre fabricated tissue engineered bone flaps and applying rhBMP 2 in situ to repair segmental, mandibular critical size defects. From animal experiments reported by Boyne [32] and Seto et al. [33], the width of the mandibular "critical size" defect in the Rhesus monkey was $20 \mathrm{~mm}$. Therefore, we did not include a control group in our study. The sample size in each group was limited because of the expensive animal model, but our study provides a basis for future study and clinical application.

\section{Conclusions}

We validated that a prefabricated tissue engineered bone flap can be used with rhBMP 2 incorporated DFDBA and CHA scaf folds implanted in Rhesus monkey latissimus dorsi muscle for mandibular reconstruction. The pedicled prefabricated bone flaps showed better bone regeneration than did rhBMP 2 incorporated DFDBA and CHA scaffolds alone in mandibular reconstruction. The segmental mandibular defects could not be repaired with DFDBA and CHA implanted without rhBMP 2 in situ. Our results indicate the feasibility of the use of prefabricated tissue engi neered bone flap for repair of large, compound mandibular defects in the clinic.

\section{Acknowledgements}

This project was financially supported by the Beijing Municipal Commission of Science and Technology (D090600704040291). We thank the Ear, Nose and Throat Research Institute, Department of Stomatology, Department of Nuclear Medicine and Animal Center of Chinese PLA General Hospital for their generous help. We thank JIMAFEI Science and Technology Development Co. Ltd. for manu facture custom titanium meshes. We thank OsteoRad Biomaterial Co. Ltd. (China) for manufacture the DFDBA used in the study.

\section{Appendix}

Figures with essential colour discrimination. Most of the figures in this article have parts that are difficult to interpret in black and white. The full colour images can be found in the on line version, at doi:10.1016/j.biomaterials.2010.02.072.

\section{References}

[1] Goh BT, Lee S, Tideman H, Stoelinga PJ. Mandibular reconstruction in adults: a review. Int J Oral Maxillofac Surg 2008;37(7):597 605.

[2] Spector JA, Warren SM, Singh SP, McCarthy JG, Siebert JW. Marriage of hard and soft tissues of the face revisited: when distraction meets microsurgery. Ann Plast Surg 2007;59(1):1 5.
[3] Warnke PH, Wiltfang J, Springer I, Acil Y, Bolte H, Kosmahl M, et al. Man as living bioreactor: fate of an exogenously prepared customized tissue-engineered mandible. Biomaterials 2006;27(17):3163 7.

[4] Warnke PH, Springer IN, Wiltfang J, Acil Y, Eufinger H, Wehmoller M, et al Growth and transplantation of a custom vascularised bone graft in a man. Lancet 2004;364(9436):766 70 .

[5] Yuan J, Cui L, Zhang WJ, Liu W, Cao Y. Repair of canine mandibular bone defects with bone marrow stromal cells and porous beta-tricalcium phosphate. Biomaterials 2007;28(6):1005 13.

[6] Bessa PC, Casal M, Reis RL. Bone morphogenetic proteins in tissue engineering: the road from the laboratory to the clinic, part I (basic concepts). J Tissue Eng Regen Med 2008;2(1):1 13.

[7] Bessa PC, Casal M, Reis RL. Bone morphogenetic proteins in tissue engineering the road from laboratory to clinic, part II (BMP delivery). J Tissue Eng Regen Med 2008;2(2 3):81 96.

[8] Herford AS, Boyne PJ. Reconstruction of mandibular continuity defects with bone morphogenetic protein-2 (rhBMP-2). J Oral Maxillofac Surg 2008;66 (4):616 24.

[9] Clokie CM, Sandor GK. Reconstruction of 10 major mandibular defects using bioimplants containing BMP-7. J Can Dent Assoc 2008;74 (1):67 72 .

[10] Heliotis M, Lavery KM, Ripamonti U, Tsiridis E, di Silvio L. Transformation of a prefabricated hydroxyapatite/osteogenic protein-1 implant into a vascularised pedicled bone flap in the human chest. Int J Oral Maxillofac Surg 2006;35(3):265 9.

[11] Seto I, Tachikawa N, Mori M, Hoshino S, Marukawa E, Asahina I, et al. Restoration of occlusal function using osseointegrated implants in the canine mandible reconstructed by rhBMP-2. Clin Oral Implants Res 2002;13 (5):536 41.

[12] Terheyden $\mathrm{H}$, Jepsen S, Rueger DR. Mandibular reconstruction in miniature pigs with prefabricated vascularized bone grafts using recombinant human osteogenic protein-1: a preliminary study. Int J Oral Maxillofac Surg 1999;28 (6):461 3 .

[13] Terheyden H, Knak C, Jepsen S, Palmie S, Rueger DR. Mandibular reconstruction with a prefabricated vascularized bone graft using recombinant human osteogenic protein-1: an experimental study in miniature pigs. Part I: prefabrication. Int J Oral Maxillofac Surg 2001;30(5):373 9.

[14] Seeherman H, Wozney JM. Delivery of bone morphogenetic proteins for orthopedic tissue regeneration. Cytokine Growth Factor Rev 2005;16 (3):329 45.

[15] Li RH, Wozney JM. Delivering on the promise of bone morphogenetic proteins. Trends Biotechnol 2001;19(7):255 65

[16] Hahn M, Vogel M, Delling G. Undecalcified preparation of bone tissue: report of technical experience and development of new methods. Virchows Arch A Pathol Anat Histopathol 1991;418(1):1 7.

[17] Rouwkema J, Rivron NC, van Blitterswijk CA. Vascularization in tissue engineering. Trends Biotechnol 2008;26(8):434 41.

[18] Hollister SJ. Porous scaffold design for tissue engineering. Nat Mater 2005 $4(7): 51824$

[19] Zhou J, Lin H, Fang T, Li X, Dai W, Uemura T, et al. The repair of large segmental bone defects in the rabbit with vascularized tissue engineered bone. Biomaterials 2010;31(6):1171 9.

[20] Richardson TP, Peters MC, Ennett AB, Mooney DJ. Polymeric system for dua growth factor delivery. Nat Biotechnol 2001;19(11):1029 34.

[21] Santos MI, Reis RL. Vascularization in bone tissue engineering: physiology, current strategies, major hurdles and future challenges. Macromol Biosci 2010;10(1):12 27

22] Celeste AJ, Iannazzi JA, Taylor RC, Hewick RM, Rosen V, Wang EA, et al. Identification of transforming growth factor beta family members present in bone-inductive protein purified from bovine bone. Proc Natl Acad Sci USA 1990;87(24):9843 7.

[23] Mikulski AJ, Urist MR. Collagenase-released non-collagenous proteins of cortical bone matrix. Prep Biochem 1977;7(5):357 81

[24] Boyne PJ. Application of bone morphogenetic proteins in the treatment of clinical oral and maxillofacial osseous defects. J Bone Joint Surg Am 2001;83-A (Suppl. 1 (Pt 2)):S146 50.

[25] Deckers MM, van Bezooijen RL, van der Horst G, Hoogendam J, van Der Bent C, Papapoulos SE, et al. Bone morphogenetic proteins stimulate angiogenesis through osteoblast-derived vascular endothelial growth factor A. Endocrinology 2002;143(4):1545 53.

[26] Terheyden H, Warnke P, Dunsche A, Jepsen S, Brenner W, Palmie S, et al Mandibular reconstruction with prefabricated vascularized bone grafts using recombinant human osteogenic protein-1: an experimental study in miniature pigs. Part II: transplantation. Int J Oral Maxillofac Surg 2001;30(6): 46978.

27] Chen B, Lin H, Wang J, Zhao Y, Wang B, Zhao W, et al. Homogeneous osteogenesis and bone regeneration by demineralized bone matrix loading with collagen-targeting bone morphogenetic protein-2. Biomaterials 2007;28 (6):1027 35

[28] Aspenberg P, Wang E, Thorngren KG. Bone morphogenetic protein induces bone in the squirrel monkey, but bone matrix does not. Acta Orthop Scand 1992;63(6):619 22

[29] Aspenberg P, Turek T. BMP-2 for intramuscular bone induction: effect in squirrel monkeys is dependent on implantation site. Acta Orthop Scand 1996; 67(1):3 6 . 
[30] Aspenberg P, Lohmander LS, Thorngren KG. Monkey bone matrix induces bone formation in the athymic rat, but not in adult monkeys. J Orthop Res 1991;9(1):20 5 .

[31] Paul BF, Horning GM, Hellstein JW, Schafer DR. The osteoinductive potential of demineralized freeze-dried bone allograft in human non-orthotopic sites: a pilot study. J Periodontol 2001;72(8):1064 8.
[32] Boyne PJ, Salina S, Nakamura A, Audia F, Shabahang S. Bone regeneration using rhBMP-2 induction in hemimandibulectomy type defects of elderly subhuman primates. Cell Tissue Bank 2006;7(1):1 10.

[33] Seto I, Asahina I, Oda M, Enomoto S. Reconstruction of the primate mandible with a combination graft of recombinant human bone morphogenetic protein-2 and bone marrow. J Oral Maxillofac Surg 2001;59(1):53 61 [discussion 2 3].

Please cite this article in press as: Zhou M, et al., Primate mandibular reconstruction with prefabricated, vascularized tissue engineered bone... Biomaterials (2010), doi:10.1016/j.biomaterials.2010.02.072 